\title{
MicroRNA expression profile of a Malaysian Bajau family with familial mitochondrial neurogastrointestinal encephalomyopathy
}

\author{
F.L. Yong ${ }^{1}$, C.W. Wang ${ }^{2}$ and K.S. Tan ${ }^{1}$ \\ ${ }^{1}$ Department of Medicine, Faculty of Medicine, University of Malaya, \\ Kuala Lumpur, Malaysia \\ ${ }^{2}$ Department of Biochemistry, Faculty of Medicine, MAHSA University, \\ Kuala Lumpur, Malaysia \\ Corresponding author: K.S. Tan \\ E-mail: tanks@ummc.edu.my \\ Genet. Mol. Res. 14 (4): 13172-13183 (2015) \\ Received May 11, 2015 \\ Accepted July 24, 2015 \\ Published October 26, 2015 \\ DOI http://dx.doi.org/10.4238/2015.October.26.13
}

ABSTRACT. Mitochondrial neurogastrointestinal encephalomyopathy (MNGIE) is a rare, autosomal recessive disorder associated with mutations in the thymidine phosphorylase (TYMP) gene. The main objective of this study was to characterize the genetic profiles of the deceased proband's family members $(\mathrm{N}=4)$ using DNA sequencing and to determine miRNA deregulation in MNGIE using miRNA microarray profiling and bioinformatic analysis. We found that the genetic profile of the younger sister showed similar TYMP gene mutations as that of the proband with the exception of a heterozygous mutation in exon 10. The miRNA microarray revealed 55 significantly up-regulated and 65 significantly down-regulated miRNAs. These miRNAs have been implicated in various mitochondrial dynamics such as energy metabolism, Krebs cycle, mitochondria-associated apoptosis, and mitophagy. In conclusion, we demonstrate that blood miRNAs are deregulated in the pathogenesis of MNGIE and these changes may have therapeutic implications. Further experimental studies will be required to elucidate the functional miRNA-mRNA interactions in MNGIE.

Key words: Bajau; microRNA; miRWalk; MNGIE; TYMP 


\section{INTRODUCTION}

Mitochondrial neurogastrointestinal encephalomyopathy (MNGIE) is a rare, autosomal recessive disorder caused by mutations in the thymidine phosphorylase (TYMP) gene, which catalyzes the breakdown of pyrimidine nucleosides. Excessive thymidine accumulation alters mitochondrial nucleotide and nucleoside pools, resulting in impaired mitochondrial DNA repair and replication (Taylor and Turnbull, 2005). Clinically, MNGIE presents as a multisystem disorder with polyneuropathy, ophthalmoplegia, leukoencephalopathy, and intestinal pseudo-obstruction during the second and fifth decades of life (Papadimitriou et al., 1998). The TYMP gene is located on chromosome 22q13 (Slama et al., 2005). TYMP gene defect generally leads to the loss of TYMP enzyme activity that catabolizes the phosphorolysis of thymidine to thymine and 2-deoxy-D-ribose 1-phosphate (Nishino et al., 2000; Tan et al., 2012). The TYMP enzyme is also known as endothelial cell growth factor 1 (ECGF1) (Slager et al., 2006). It is a vital enzyme that is widely expressed in the tissues of the brain, peripheral nerves, gastrointestinal system, lung, and bladder (Yoshimura et al., 1990). Besides having a catalytic function, the TYMP enzyme is implicated in angiogenesis and cell trophism processes in cancer (Nishino et al., 2000). To date, approximately 30 TYMP mutations have been identified in homozygous or compound heterozygous MNGIE patients (Kintarak et al., 2007). MNGIE diagnosis relies on clinical manifestations, biochemical correlates of TYMP enzyme activity, levels of thymidine and deoxyuridine as well as genetic testing (Szigeti et al., 2004). However, the major diagnostic hurdle is characterized by non-specific neurological and gastrointestinal symptoms that may be mistaken as psychiatric or gastrointestinal disorders (Garone et al., 2011). The mean age of death is 37 years due to complications such as intestinal rupture and chronic pseudo-obstruction, cachexia, peritonitis, esophageal bleeding, cirrhosis, and aspiration pneumonia (Szigeti et al., 2004; Tan et al., 2012). To date, there is no definitive treatment for MNGIE. Several treatment options to prolong patient's survival include allogeneic hematopoietic stem cell transplantation, exogenous TYMP enzyme replacement therapy and measures to stabilize plasma thymidine and deoxyuridine (Garone et al., 2011).

In recent years, microRNAs (miRNAs) have been found to be involved in the pathogenesis of various diseases. miRNAs are short, non-coding RNA molecules of 19-22 nucleotides that act as regulators of gene expression (Bartel, 2004). The mechanism of action is through complementarity binding between the seed region of miRNAs and the 3'-untranslated region (3'-UTR) of target mRNAs (Bagga et al., 2005). miRNAs have been described to modulate important signaling pathways in the mitochondria such as cellular energy metabolism, Krebs cycle, mitochondriaassociated apoptosis, and mitophagy (Bienertova-Vasku et al., 2013). While the involvement of miRNAs in the pathophysiology of several mitochondrial DNA diseases have been discussed, miRNA regulation in MNGIE have not been previously described (Chinnery et al., 2012).

We reported the first MNGIE case of a Malaysian patient of Bajau descent in Sabah, Malaysia, which was confirmed by DNA sequencing (Tan et al., 2012). A study of the family members was undertaken with additional objectives. The objectives were to characterize the genetic profiles of the proband's direct family members and to elucidate miRNA regulation in this condition by microarray profiling and analysis.

\section{MATERIAL AND METHODS}

\section{Study design}

A case-control study was designed to determine the genetic profiles and the differentially 
expressed miRNAs in the whole blood samples of a Bajau family directly related to the first MNGIE patient in Malaysia (proband) (Figure 1). This study was approved by the Medical Ethics Committee of University of Malaya Medical Centre (UMMC) (reference number 878.11). Whole blood samples were collected from the deceased proband's immediate family members. The family characteristics are summarized in Figure 1. Twelve normal, age-, and sex-matched healthy controls from the Bajau ethnic group were selected. Written informed consent was obtained from each participant. The whole blood samples were collected in ethylenediamine tetraacetic acid (EDTA) tubes.

I

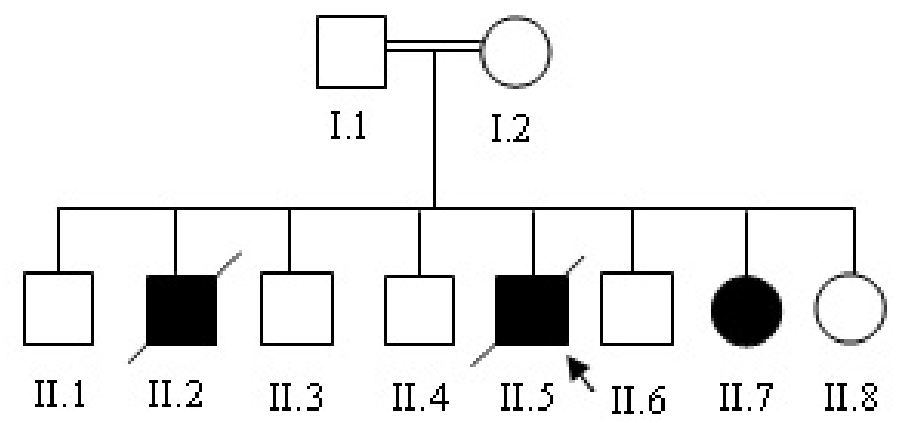

Figure 1. Genetic pedigree of the Bajau family with MNGIE.

\section{DNA isolation and direct sequencing}

DNA from whole blood was isolated using QIAamp DNA Blood Mini Kit (Qiagen, Venlo, Limburg, Netherlands) according to the manufacturer protocol. The nine coding exons of the TYMP gene (exons 2-10) were amplified using previously published primers (Slama et al., 2005; Kintarak et al., 2007). Direct sequencing of both sense and antisense strands of the amplified products was performed using dye terminator chemistry as described previously (Tan et al., 2012).

\section{Total RNA isolation}

Total RNA (including miRNAs) from whole blood was isolated using Ribopure Blood RNA Isolation Kit (Ambion, Carlsbad, CA, USA) according to the manufacturer instructions. RNA concentration and integrity were determined using a NanoDrop 2000 spectrophotometer (Thermo Scientific Wilmington, DE, USA) and Agilent 2100 Bioanalyzer (Agilent Technologies, Santa Clara, CA, USA).

\section{miRNA microarray}

The miRNA expression profile was generated by GeneChip miRNA 3.0 Array (Affymetrix, Santa Clara, CA, USA). The array contains 19,724 probe sets with 1,733 of them detecting human mature miRNAs. The content is derived from miRBase Release 17. The miRNA expression profiling analysis for the deceased proband's father, mother, fourth elder brother, and seventh younger sister was conducted individually whereas the profiling analysis for the controls was performed in four replicates, with $\mathrm{N}=3$ each.

Briefly, total RNA $(1 \mu \mathrm{g})$ was biotin-labeled using Affymetrix Flashtag Biotin HSR RNA Labeling Kit and hybridized onto the array chip for $16 \mathrm{~h}$ in Affymetrix Hybridization Oven 640. The chip was washed and stained using Affymetrix Fluidics Station 450 and scanned with 
GeneChip Scanner 3000 7G. The cell intensity file was generated and analyzed using Affymetrix Transcriptome Analysis Console 2.0 Software. The normalized data were analyzed using ANOVA analysis $(P<0.05)$ and filtered at a fold change cut-off of 1.5 . Hierarchical clustering was performed using similarity measure of Euclidean distance and complete linkage method and expressed in a heatmap and principal component analysis (PCA) plot.

\section{In silico target prediction}

In silico target prediction between miRNAs and TYMP gene was carried out via the miRWalk database (http://www.umm.uni-heidelberg.de/apps/zmf/mirwalk) (Dweep et al., 2011).

\section{Reverse transcription-quantitative real time PCR (RT-qPCR)}

Reverse transcription for miRNAwas performed using TaqMan MicroRNAReverse Transcription Reagents (Applied Biosystems, Carlsbad, CA, USA). qPCR was conducted on StepOnePlus Real Time PCR system using TaqMan MicroRNA Assay and TaqMan Fast Advanced Master Mix. RNU48 was chosen as the endogenous control. All assays were performed in triplicate based on the manufacturer protocol. The relative expression was determined using comparative CT $\left(2^{-\Delta \Delta c t}\right)$ method (Yuan et al., 2006). Statistical significance was determined by unpaired $t$-test, with $P<0.05$. Statistical analysis was performed using SPSS version 16.0 software (IBM Corporation, Armonk, NY).

\section{RESULTS}

\section{Genetic study}

The genetic profiling of the deceased proband (II.5) was conducted previously (Tan et al., 2012). Since MNGIE is a familial disorder, the present study was designed to characterize the genetic and miRNA profiles of the proband's family members. However, some of the family members (II.1, II.2, II.3, II.6, and II.8) did not respond to our participation request in the present study. Hence, the genetic profiling studies were limited to only four family members, consisting of the deceased proband's father (I.1), mother (I.2), fourth elder brother (II.4), and seventh younger sister (II.7) (Tables 1 and 2). The genetic profiles of the family members indicated that the younger sister (II.7) presents similar mutations with the deceased proband, except for a heterozygous mutation in exon 10. Her clinical presentation was consistent with MNGIE.

\section{miRNA microarray study}

Blood miRNA profiling analysis of the deceased proband's father (I.1), mother (I.2), fourth elder brother (II.4), and seventh younger sister (II.7) was conducted. However, the blood sample from the deceased proband needed for miRNA analysis was not obtained. The heatmap in Figure $2 \mathrm{~A}$ indicates the number of miRNAs that were differentially regulated between the Bajau family with familial MNGIE and the control group. At the fold change cut-off of 1.5, the miRNA microarray revealed 55 significantly up-regulated and 65 significantly down-regulated miRNAs (Table 3). Next, a PCA plot was computed to provide a visual representation of the samples. The PCA plot in Figure $2 \mathrm{~B}$ shows that the samples were distributed in a distinctive pattern, based on the variance in miRNA gene expression. 
F.L. Yong et al.

13176

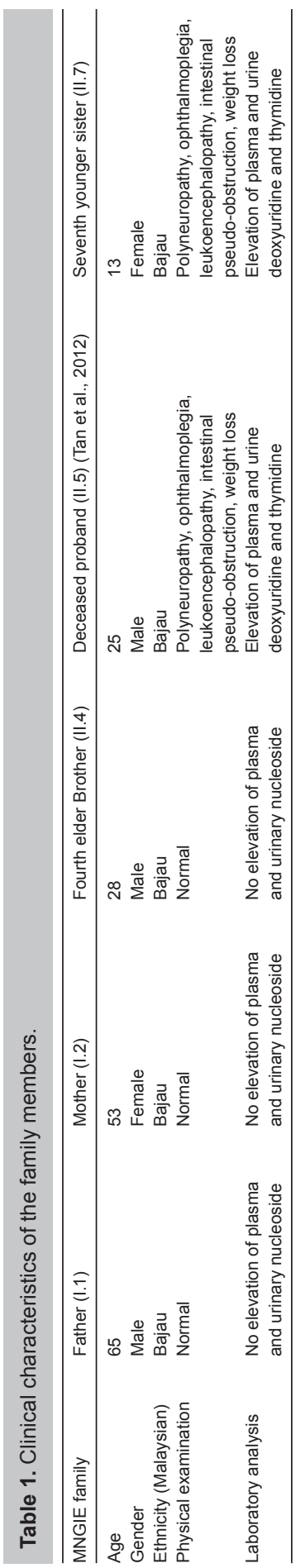

Genetics and Molecular Research 14 (4): $13172-13183$ (2015) 


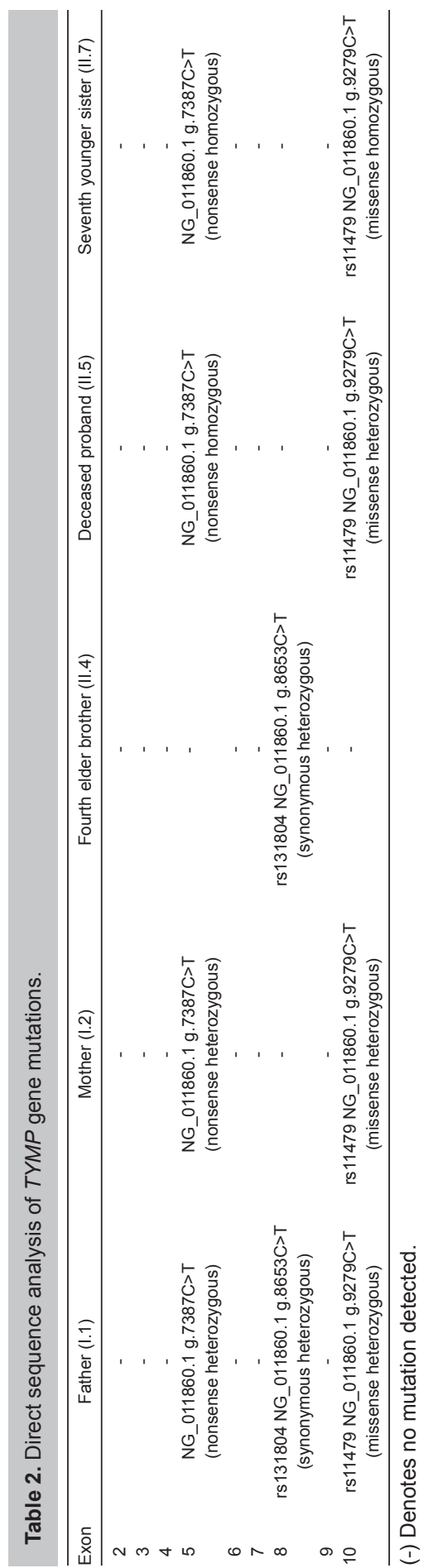


Table 3. List of differentially expressed miRNAs among the family with MNGIE disorder and healthy controls.

\begin{tabular}{|c|c|c|c|}
\hline Up-regulated miRNA & Fold change & Down-regulated miRNA & Fold change \\
\hline hsa-miR-10b-star_st & 1.54 & hsa-miR-101_st & -3.65 \\
\hline hsa-miR-1228-star_st & 1.91 & hsa-miR-128_st & -1.5 \\
\hline hsa-miR-1268_st & 1.64 & hsa-miR-1284_st & -1.99 \\
\hline hsa-miR-1288_st & 1.77 & hsa-miR-138_st & -2.67 \\
\hline hsa-miR-149-star_st & 1.79 & hsa-miR-139-5p_st & -1.77 \\
\hline hsa-miR-1587_st & 2.16 & hsa-miR-140-5p_st & -2.26 \\
\hline hsa-miR-1908_st & 1.97 & hsa-miR-146b-5p_st & -1.84 \\
\hline hsa-miR-1915_st & 1.59 & hsa-miR-148a_st & -2.85 \\
\hline hsa-miR-193a-5p_st & 2.28 & hsa-miR-148b_st & -2.7 \\
\hline hsa-miR-202_st & 1.52 & hsa-miR-181a-2-star_st & -1.75 \\
\hline hsa-miR-214_st & 1.58 & hsa-miR-183_st & -1.7 \\
\hline hsa-miR-2861_st & 1.5 & hsa-miR-183-star_st & -1.52 \\
\hline hsa-miR-3152-3p_st & 1.57 & hsa-miR-192-star_st & -4.1 \\
\hline hsa-miR-3175_st & 1.65 & hsa-miR-199a-3p_st & -2.57 \\
\hline hsa-miR-3196_st & 1.72 & hsa-miR-199a-5p_st & -2.12 \\
\hline hsa-miR-3656_st & 1.51 & hsa-miR-199b-3p_st & -2.2 \\
\hline hsa-miR-3937_st & 1.52 & hsa-miR-19a_st & -2.5 \\
\hline hsa-miR-3940-5p_st & 1.68 & hsa-miR-19b-1-star_st & -1.7 \\
\hline hsa-miR-4259_st & 1.6 & hsa-miR-20b-star_st & -1.68 \\
\hline hsa-miR-4270_st & 1.56 & hsa-miR-212_st & -1.53 \\
\hline hsa-miR-4281_st & 1.88 & hsa-miR-221-star_st & -3.02 \\
\hline hsa-miR-4433_st & 1.59 & hsa-miR-27b_st & -2.05 \\
\hline hsa-miR-4436a_st & 1.71 & hsa-miR-27b-star_st & -2.19 \\
\hline hsa-miR-4450_st & 1.7 & hsa-miR-2964a-5p_st & -1.72 \\
\hline hsa-miR-4458_st & 1.51 & hsa-miR-29a_st & -2.44 \\
\hline hsa-miR-4459_st & 2.06 & hsa-miR-29b_st & -2.65 \\
\hline hsa-miR-4463_st & 1.7 & hsa-miR-29c_st & -2.66 \\
\hline hsa-miR-4466_st & 1.52 & hsa-miR-29c-star_st & -3.42 \\
\hline hsa-miR-4492_st & 2.18 & hsa-miR-301a_st & -2.62 \\
\hline hsa-miR-4502_st & 1.52 & hsa-miR-30c_st & -1.8 \\
\hline hsa-miR-4505_st & 2.18 & hsa-miR-30e_st & -1.81 \\
\hline hsa-miR-4507_st & 1.83 & hsa-miR-31_st & -2.64 \\
\hline hsa-miR-4508_st & 1.64 & hsa-miR-3130-5p_st & -4.72 \\
\hline hsa-miR-4510_st & 1.62 & hsa-miR-3145-5p_st & -2.19 \\
\hline hsa-miR-4512_st & 1.51 & hsa-miR-3158-3p_st & -1.6 \\
\hline hsa-miR-4514_st & 1.55 & hsa-miR-3165_st & -1.93 \\
\hline hsa-miR-4516_st & 1.52 & hsa-miR-3195_st & -2.15 \\
\hline hsa-miR-4524_st & 1.93 & hsa-miR-326_st & -2.1 \\
\hline hsa-miR-4530_st & 1.8 & hsa-miR-330-5p_st & -2.09 \\
\hline hsa-miR-4534_st & 1.99 & hsa-miR-33b-star_st & -1.67 \\
\hline hsa-miR-4651_st & 1.66 & hsa-miR-3680-star_st & -2.53 \\
\hline hsa-miR-4669_st & 2.2 & hsa-miR-3688-3p_st & -1.83 \\
\hline hsa-miR-4687-3p_st & 1.7 & hsa-miR-374a_st & -1.87 \\
\hline hsa-miR-4690-5p_st & 2.52 & hsa-miR-374b_st & -2.28 \\
\hline hsa-miR-4695-5p_st & 1.57 & hsa-miR-376c_st & -2.8 \\
\hline hsa-miR-4710_st & 1.86 & hsa-miR-3940-3p_st & -1.53 \\
\hline hsa-miR-4732-5p_st & 1.65 & hsa-miR-4286_st & -1.62 \\
\hline hsa-miR-4734_st & 1.93 & hsa-miR-454_st & -1.6 \\
\hline hsa-miR-4763-3p_st & 1.59 & hsa-miR-487a_st & -3.73 \\
\hline hsa-miR-4774-5p_st & 1.57 & hsa-miR-495_st & -1.59 \\
\hline hsa-miR-486-3p_st & 3.47 & hsa-miR-502-5p_st & -2.27 \\
\hline hsa-miR-638_st & 1.56 & hsa-miR-505_st & -2.67 \\
\hline hsa-miR-762_st & 1.8 & hsa-miR-589_st & -1.74 \\
\hline hsa-miR-885-3p_st & 1.64 & hsa-miR-590-5p_st & -2.33 \\
\hline \multirow[t]{11}{*}{ hsa-miR-939_st } & 1.61 & hsa-miR-616_st & -1.81 \\
\hline & & hsa-miR-624-star_st & -3.09 \\
\hline & & hsa-miR-627_st & -1.69 \\
\hline & & hsa-miR-636_st & -1.79 \\
\hline & & hsa-miR-641_st & -1.59 \\
\hline & & hsa-miR-643_st & -1.94 \\
\hline & & hsa-miR-647_st & -1.73 \\
\hline & & hsa-miR-7_st & -2.06 \\
\hline & & hsa-miR-7-1-star_st & -2.58 \\
\hline & & hsa-miR-96_st & -2.4 \\
\hline & & hsa-miR-99a_st & -2.24 \\
\hline
\end{tabular}


A

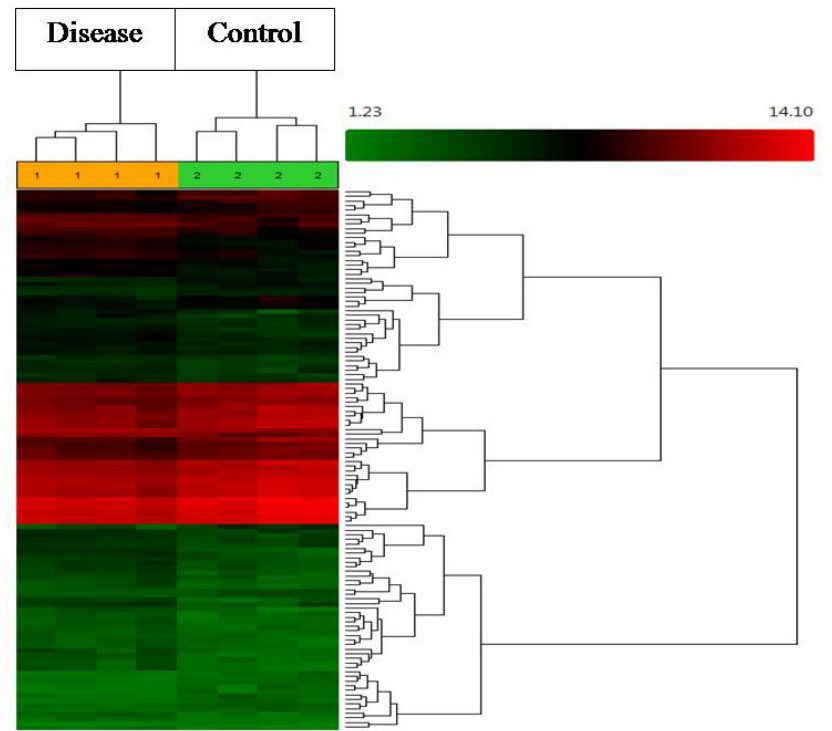

B

PCA - Probe Cell Intensity Data (PCA Mapping 90.7\%)

\section{PCA2 11.7\%}

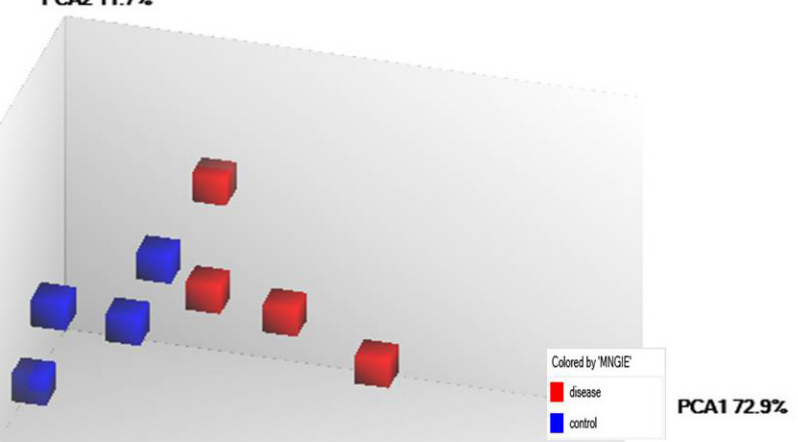

PCA3 6.1\%

Figure 2. (A) Heatmap and (B) PCA plot of the Bajau family with MNGIE.

\section{In silico prediction and RT-qPCR study}

Based on the miRWalk database, only two miRNAs, namely miR-28-5p and miR-708 were predicted to target the TYMP gene. However, there was no overlapped between the list of miRNAs obtained in our miRNA microarray analysis and the in silico miRNA prediction. Nevertheless, the two putative miRNAs were still selected for RT-qPCR validation (Figure 3A and B). Unfortunately, no statistical significance was achieved $(P>0.05)$. The results showed that the predicted miR-28$5 p$ and miR-708 were not differentially expressed in this Bajau family with familial MNGIE. 
$\mathbf{A}$

miR-28-5p

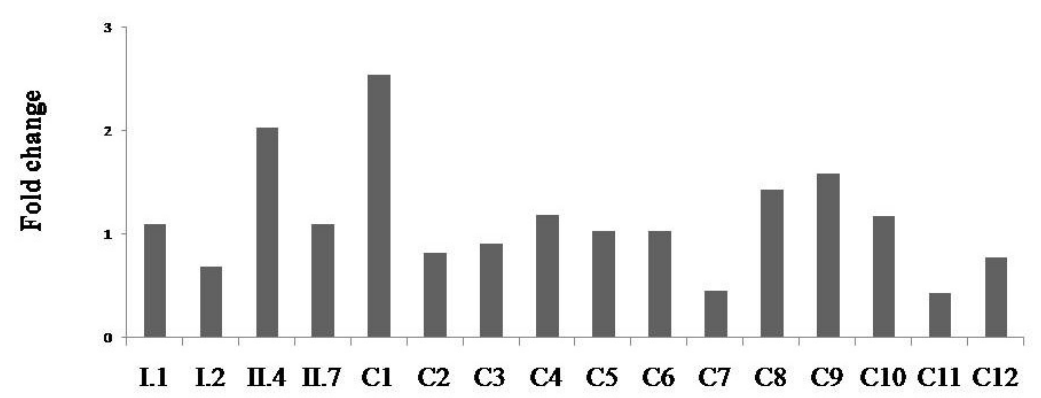

B

miR-708

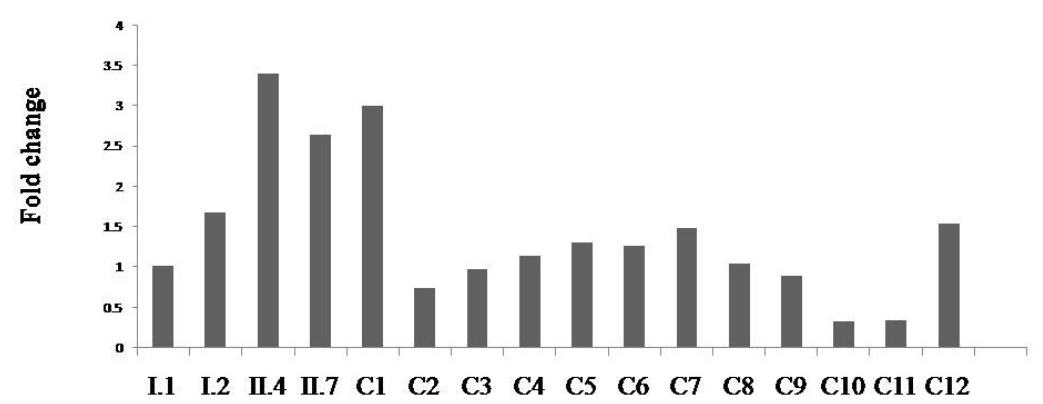

Figure 3. RT-qPCR analysis of (A) miR-28-5p and (B) miR-708. No statistical significance was observed. Column I.1 = father; Column I.2 = mother; Column II.4 = fourth elder brother; Column II.7 = seventh younger sister; Columns C1C12 $=$ healthy control.

\section{DISCUSSION}

This paper is the first description of miRNA profiling from a family with two clinically affected members with MNGIE. The focus of the present study was to characterize the genetic mutations found in the coding exon 2-10 of the TYMP gene among the family members of the first reported case of MNGIE disorder in Sabah, Malaysia. The proband and his siblings were the product of a consanguineous marriage as both parents were distant cousins. This family was part of an indigenous Bajau tribe of East Malaysia. As an ethnic group that originated many generations ago from the Southern Philippines (Ali, 2010), the Bajau tribe is currently the second largest ethnic group after the Kadazans in Sabah, forming 13.4\% of the population (Sabah Tourist Association, 2015). The result of consanguineous marriages is the high incidence of genetic mutations due to homozygosity as observed in MNGIE (Tan et al., 2012). Among the deceased proband's father (I.1), mother (I.2), and elder brother (II.4), only the younger sister (II.7) was clinically and biochemically diagnosed with MNGIE. She possesses similar mutations with the deceased proband, with a heterozygous mutation in exon 10 . The main limitation of the present study was the inability to obtain blood samples needed for miRNA analysis from the proband and the other five siblings. The proband and his second elder brother (II.2) had passed away shortly after being diagnosed with MNGIE. The remaining four healthy asymptomatic siblings refused to participate in this project. 
Based on the list of miRNAs obtained through our microarray analysis, highly differentially regulated miRNAs included miR-486-3p, miR-221*, miR-624*, miR-29c*, miR-101, miR-487a, miR-192*, and miR-3130-5p. These miRNAs were depicted with fold change values of at least 3.00 (Table 3). miR-486-3p and miR-624* have been implicated in vascular hematopoiesis and homeostasis (Lulli et al., 2013; Tijsen et al., 2012). miR-192* is a unique miRNA for kidney diseases such as nephropathy and renal fibrosis (Chung et al., 2010). miR-221*, miR-29c*, miR-101, miR487a, and miR-3130-5p have been involved in cellular proliferation and differentiation (le Sage et al., 2007; Strillacci et al., 2009; Pass et al., 2010; Berillo et al., 2013; Ma et al., 2013). On the other hand, numerous mitochondrial miRNAs have been identified from our microarray analysis. Although these miRNAs have not been reported in the MNGIE disorder, their involvement in mitochondrial modulation has been described. For instance, the deregulation of miR-149* and miR-27b is implicated in mitochondrial dysfunction of the skeletal and adipose tissues, respectively (Kang et al., 2013; Mohamed et al., 2014). miR-183 is involved in the regulation of energy metabolism in Krebs cycle (Bienertova-Vasku et al., 2013). In terms of mitochondria catabolic metabolism, miR101 plays an important role in targeting the transcripts of autophagy-related proteins, while miR$221^{*}$ is involved in cell senescence (Frankel et al., 2011; Rippo et al., 2014).

In this study, the in silico miRNA prediction was computed using miRWalk, a database that integrates several miRNA target prediction programs: DIANA-MicroT, miRanda, miRWalk, MirTarget2/miRDB, PicTar, RNA22, RNAhybrid, and TargetScan Human (Table 4). Each of these algorithms has its own prediction of false positive and false negative. According to Min and Yoon (2010), the reliable practice for a good miRNA-mRNA gene prediction involves the use of three to five algorithms. The unique feature of the miRWalk database is its ability to provide a score of overlap from the eight algorithms (Dweep et al., 2011). However, the in silico analysis in the present study yielded only two miRNAs that could target the TYMP gene, namely miR-28-5p and miR-708. The individual score of overlap for both miRNAs was low, which was only two (miRanda and miRWalk) out of the eight algorithms. To our knowledge, this is the first study on the characterization of potential linkage between miRNAs and the TYMP gene in MNGIE. The miRNA microarray and the RT-qPCR validation results indicated that the predicted miR-28-5p and miR-708 were not significantly regulated. These two miRNAs may not be involved in the regulation of the TYMP gene, as opposed to the prediction obtained through the miRWalk database. To date, no single computational method could accurately predict miRNAs that target the TYMP gene in MNGIE. It is a challenging task to predict specific miRNA-mRNA interaction due to the partial complementarity of binding between the seed region of miRNA and the 3'-UTR of target mRNA (Rehmsmeier et al., 2004). The common principles governing the binding capability are base pairing pattern, thermodynamic stability of miRNA-mRNA hybrid, comparative sequence analysis, determination of multiple targets sites per target transcript, and statistical evaluations (Min and Yoon, 2010; Sotillo and Thomas-Tikhonenko, 2011).

\begin{tabular}{|c|c|c|c|c|}
\hline Program & Recommended target species & Algorithm & Web address & Reference \\
\hline DIANA-microT & Any species & Thermodynamics & http://diana.cslab.ece.ntua.gr/microT/ & (Maragkakis et al., 2009) \\
\hline miRanda & Flies, vertebrates & Seed complementarity & http://www.microrna.org/microrna/home.do & (Enright et al. 2003) \\
\hline miRWalk & Humans, mice, rats & Seed complementarity & http://www.umm.uni-heidelberg.de/apps/zmf/mirwalk/ & (Dweep et al., 2011) \\
\hline MirTarget2/miRDB & $\begin{array}{l}\text { Humans, mice, rats, } \\
\text { dogs, chickens }\end{array}$ & Support vector machine classifier & http://mirdb.org/miRDB/ & (Wang, 2008) \\
\hline PicTar & Vertebrates, flies, worms & Thermodynamics & http://www.pictar.org/ & (Krek et al., 2005) \\
\hline RNA22 & Any species & Pattern recognition & http://cbcsrv.watson.ibm.com/rna22.html & (Miranda et al., 2006) \\
\hline RNAhybrid & Any species & Thermodynamics, statistical model & http://bibiserv.techfak.uni-bielefeld.de/rnahybrid/ & (Rehmsmeier et al., 2004) \\
\hline TargetScan/TargetScanS & Vertebrates & Seed complementarity & http://www.targetscan.org/ & $\begin{array}{l}\text { (Lewis et al., 2003; } \\
\text { Lewis et al., 2005) }\end{array}$ \\
\hline
\end{tabular}




\section{CONCLUSIONS}

In conclusion, the genetic profiles of the four family members with MNGIE indicate that the younger sister of the proband possessed similar TYMP gene mutations with the exception of a heterozygous mutation in exon 10. No significant association was found between the TYMP gene and the 120 differentially expressed miRNAs obtained from our miRNA microarray study. Our work highlights the importance of further investigation in more cases of MNGIE patients and their family members. Moreover, there is a need to develop more accurate computational algorithms for the identification of functional miRNA-mRNA interactions.

\section{ACKNOWLEDGMENTS}

The authors thank Dr Lian Lay Hoong and Noreena Mohd Nor for their expert opinion in the genetic analysis. The study was funded by the University of Malaya (Malaysia) High Impact Research Grant (UM.C/625/1/HIR/ MOHE/MED/08-E000036).

\section{REFERENCES}

Ali I (2010). Since birth till death, what is their status: a case study of the sea bajau in pulau mabul, Semporna. J. Arts Sci. Commer. 1: 156-165.

Bagga S, Bracht J, Hunter S, Massirer K, et al. (2005). Regulation by let-7 and lin-4 miRNAs results in target mRNA degradation. Cell 122: 553-563.

Bartel DP (2004). MicroRNAs: genomics, biogenesis, mechanism, and function. Cell 116: 281-297.

Berillo O, Regnier M and Ivashchenko A (2013). Binding of intronic miRNAs to the mRNAs of host genes encoding intronic miRNAs and proteins that participate in tumorigenesis. Comput. Biol. Med. 43: 1374-1381.

Bienertova-Vasku J, Sana J and Slaby O (2013). The role of microRNAs in mitochondria in cancer. Cancer Lett. 336: 1-7.

Chinnery PF, Elliott HR, Hudson G, Samuels DC, et al. (2012). Epigenetics, epidemiology and mitochondrial DNA diseases. Int. J. Epidemiol. 41: 177-187.

Chung ACK, Huang XR, Meng X and Lan HY (2010). miR-192 mediates TGF-beta/Smad3-driven renal fibrosis. J. Am. Soc. Nephrol. 21: 1317-1325.

Dweep H, Sticht C, Pandey P and Gretz N (2011). miRWalk - database: prediction of possible miRNA binding sites by "walking" the genes of three genomes. J. Biomed. Inform. 44: 839-847.

Enright AJ, John B, Gaul U, Tuschl T, et al. (2003). MicroRNA targets in Drosophila. Genome Biol. 5: R1.

Frankel LB, Wen J, Lees M, Høyer-Hansen M, et al. (2011). microRNA-101 is a potent inhibitor of autophagy. EMBO J. 30 : 4628-4641.

Garone C, Tadesse S and Hirano M (2011). Clinical and genetic spectrum of mitochondrial neurogastrointestinal encephalomyopathy. Brain 134: 3326-3332.

Kang T, Lu W, Xu W, Anderson L, et al. (2013). MicroRNA-27 (miR-27) targets prohibitin and impairs adipocyte differentiation and mitochondrial function in human adipose-derived stem cells. J. Biol. Chem. 288: 34394-34402.

Kintarak J, Liewluck T, Sangruchi T, Hirano M, et al. (2007). A novel ECGF1 mutation in a Thai patient with mitochondrial neurogastrointestinal encephalomyopathy (MNGIE). Clin. Neurol. Neurosurg. 109: 613-616.

Krek A, Grün D, Poy MN, Wolf R, et al. (2005). Combinatorial microRNA target predictions. Nat. Genet. 37: $495-500$.

le Sage C, Nagel R, Egan DA, Schrier M, et al. (2007). Regulation of the p27Kip1 tumor suppressor by miR-221 and miR-222 promotes cancer cell proliferation. EMBO J. 26: 3699-3708.

Lewis BP, Shih IH, Jones-Rhoades MW, Bartel DP, et al. (2003). Prediction of mammalian microRNA targets. Cell 115: $787-798$.

Lewis BP, Burge CB and Bartel DP (2005). Conserved seed pairing, often flanked by adenosines, indicates that thousands of human genes are microRNA targets. Cell 120: 15-20.

Lulli V, Romania P, Morsilli O, Cianciulli P, et al. (2013). MicroRNA-486-3p regulates beta-globin expression in human erythroid cells by directly modulating BCL11A. PLoS One 8: e60436.

Ma MT, He M, Wang Y, Jiao XY, et al. (2013). MiR-487a resensitizes mitoxantrone (MX)-resistant breast cancer cells (MCF-7/ $M X)$ to $M X$ by targeting breast cancer resistance protein (BRCP/ABCG2). Cancer Lett. 339: 107-115. 
Maragkakis M, Alexiou P, Papadopoulos GL, Reczko M, et al. (2009). Accurate microRNA target prediction correlates with protein repression levels. BMC Bioinformatics 10: 295.

Min $\mathrm{H}$ and Yoon S (2010). Got target?: computational methods for microRNA target prediction and their extension. Exp. Mol. Med. 42: 233-244.

Miranda KC, Huynh T, Tay Y, Ang YS, et al. (2006). A pattern-based method for the identification of microRNA binding sites and their corresponding heteroduplexes. Cell 126: 1203-1217.

Mohamed JS, Hajira A, Pardo PS and Boriek AM (2014). MicroRNA-149 inhibits PARP-2 and promotes mitochondrial biogenesis via SIRT-1/PGC-1a network in skeletal muscle. Diabetes 63: 1546-1559.

Nishino I, Spinazzola A, Papadimitriou A, Hammans S, et al. (2000). Mitochondrial neurogastrointestinal encephalomyopathy: an autosomal recessive disorder due to thymidine phosphorylase mutations. Ann. Neurol. 47: 792-800.

Papadimitriou A, Comi GP, Hadjigeorgiou GM, Bordoni A, et al. (1998). Partial depletion and multiple deletions of muscle mtDNA in familial MNGIE syndrome. Neurology 51: 1086-1092.

Pass HI, Goparaju C, Ivanov S, Donington J, et al. (2010). hsa-miR-29c* is linked to the prognosis of malignant pleural mesothelioma. Cancer Res. 70: 1916-1924.

Rehmsmeier M, Steffen P, Hochsmann M and Giegerich R (2004). Fast and effective prediction of microRNA/target duplexes. RNA 10: 1507-1517.

Rippo MR, Olivieri F, Monsurrò V, Prattichizzo F, et al. (2014). MitomiRs in human inflamm-aging: a hypothesis involving miR181a, miR-34a and miR-146a. Exp. Gerontol. 56: 154-163.

Sabah Tourist Association. Available at [http://www.sta.my/index.cfm]. Accessed March 13, 2015.

Slager EH, Honders MW, van der Meijden ED, van Luxemburg-Heijs SA, et al. (2006). Identification of the angiogenic endothelial-cell growth factor-1/thymidine phosphorylase as a potential target for immunotherapy of cancer. Blood 107: 4954-4960.

Slama A, Lacroix C, Plante-Bordeneuve V, Lombès A, et al. (2005). Thymidine phosphorylase gene mutations in patients with mitochondrial neurogastrointestinal encephalomyopathy syndrome. Mol. Genet. Metab. 84: 326-331.

Sotillo E and Thomas-Tikhonenko A (2011). Shielding the messenger (RNA): microRNA-based anti cancer therapies. Pharmacol. Ther. 131: 18-32.

Strillacci A, Griffoni C, Sansone P, Paterini P, et al. (2009). MiR-101 downregulation is involved in cyclooxygenase-2 overexpression in human colon cancer cells. Exp. Cell Res. 315: 1439-1447.

Szigeti K, Wong LJ, Perng CL, Saifi GM, et al. (2004). MNGIE with lack of skeletal muscle involvement and a novel TP splice site mutation. J. Med. Genet. 41: 125-129.

Tan KS, Lee HG, Lian LH, Lu YS, et al. (2012). Mitochondrial neurogastrointestinal encephalomyopathy (MNGIE) in a Malaysian patient with a novel mutation in thymidine phosphorylase gene: a case report. Neurol. Asia 17: 163-168.

Taylor RW and Turnbull DM (2005). Mitochondrial DNA mutations in human disease. Nat. Rev. Genet. 6: 389-402.

Tijsen AJ, Pinto YM and Creemers EE (2012). Circulating microRNAs as diagnostic biomarkers for cardiovascular diseases. Am. J. Physiol. Heart Circ. Physiol. 303: H1085-H1095.

Wang X (2008). miRDB: a microRNA target prediction and functional annotation database with a wiki interface. RNA 14: 10121017.

Yoshimura A, Kuwazuru Y, Furukawa T, Yoshida H, et al. (1990). Purification and tissue distribution of human thymidine phosphorylase; high expression in lymphocytes, reticulocytes and tumors. Biochim. Biophys. Acta 1034: 107-113.

Yuan JS, Reed A, Chen F and Stewart Jr CN (2006). Statistical analysis of real-time PCR data. BMC Bioinformatics 7: 85. 